\title{
Análise de risco e otimização de recursos hídricos e retorno financeiro em nível de fazenda ${ }^{1}$
}

\author{
Bianca S. Tavares'2, João C. F. Borges Júnior ${ }^{3}$, Marcus M. Corrêa², \\ José R. S. Lima ${ }^{4} \&$ José D antas Neto ${ }^{5}$
}

\begin{abstract}
RESU MO
Este trabalho teve como objetivo analisar padrões de cultivo em uma propriedade rural, visando à otimização do uso de recursos hídricos e a maximização do retorno financeiro, para um estudo de caso no Agreste M eridional Pernambucano. 0 modelo de programação linear com horizonte de planejamento de três anos utilizado, foi implementado na planilha $\mathrm{M}$ icrosoft ${ }^{\circledR}$ Excel. Este modelo visou à maximização do valor presente líquido total do empreendimento. Para a análise de risco foram utilizadas duas técnicas complementares: a simulação de risco, procedida com o programa @Risk, e a análise de sensibilidade. No primeiro padrão de cultivo, $\mathrm{PC} 1$, com restrição hídrica mensal para irrigação de $8.500 \mathrm{~m}^{3}$, o máximo valor presente líquido total, VPLt, foi de $R \$ 8.771,10$ (ha ano-1); no segundo padrão de cultivo, PC2, com restrição hídrica mensal de $4.000 \mathrm{~m}^{3}$, o VPLt foi de $\mathrm{R} \$ 8.228,77$ (ha ano $)^{-1}$. $\mathrm{Na}$ análise de risco, observouse que os valores encontrados do VPLt foram positivos e próximos para os dois padrões de cultivo evidenciando a não expectativa de fracasso financeiro, mas com considerável redução da demanda de água para irrigação para o $\mathrm{PC} 2$, indicando o potencial da metodologia aplicada à gestão de recursos hídricos na região.
\end{abstract}

Palavras-chave: programação linear, método de Monte Carlo, valor presente líquido

\section{Risk analysis and optimization of water resources and financial return at farm level}

\begin{abstract}
This study aimed to analyze cropping patterns on a farm, aiming to optimize the use of water resources and maximization of financial return, for a case study in Southern Agreste Pernambucano. A linear programming model was used with a planning horizon of 3 years, implemented in spreadsheet $\mathrm{M} \mathrm{icrosoft}^{\mathrm{B}}$ Excel. The model aimed to maximize the total net present value of the enterprise. For risk analysis, it was two complementary techniques were used: the risk simulation, proceeded with the software @ Risk, and sensitivity analysis. In the first cropping pattern (PC1), with monthly water restriction for irrigation of $8,500 \mathrm{~m}^{3}$, the maximum total net present value (VPLt), was $R \$ 8,771.10$ (ha year) ${ }^{-1}$; in the second cropping pattern (PC2), with monthly water restriction of $4,000 \mathrm{~m}^{3}$, the VPLt was $R \$ 8,228.77$ (ha year) ${ }^{-1}$. It was observed in the risk analysis that the found values of VPLt were positive and closed for the two cropping patterns, making evident the absence of expectation of financial failure, but with considerable reduction of irrigation water demand for the PC2, pointing out the potential of the methodology applied to the water resources management in the region.
\end{abstract}

Key words: linear programming, M onte Carlo method, net present value

\footnotetext{
1 Parte da dissertação do primeiro autor, apoiada pelo CN Pq e FACEPE

2 DTR/U FRPE, Rua D om Manoel de Medeiros, s/n, Dois Irmãos, CEP: 52.171-900, Recife, PE. Fones: (81) 3220-6000; (81) 3320 6279. E-mail: biancaverve@yahoo.com.br; metri@dtr.ufrpe.br

${ }^{3}$ Campus Sete Lagoas/U FSJ, Rodovia M G 424, km 65, C. P. 56, CEP 35701-970, Sete Lagoas, M G. Fone (31) 3771 3305. E-mail: jcborges@ufsj.edu.br ${ }^{4}$ U AG/U FRPE, Av. Bom Pastor, s/n, Bairro Boa Vista, CEP 55296-901, Garanhuns, PE, Fone: (87) 3761-0882. E-mail: romualdo_solo@yahoo.com.br ${ }^{5}$ CTRN/UFCG, Av. A prígio Veloso 882, Bodocongó, Campus I, C. P. 10.078, CEP 58109-970, Campina Grande, PB. Fone: (83) 33101055. E-mail: zedantas@deag.ufcg.edu.br
} 


\section{INTRODUÇÃO}

Ao procurar estabelecer um planejamento no seu empreendimento, o agricultor deve selecionar, dentre as alternativas de produção, a mais eficiente na utilização dos recursos produtivos e a que satisfaz a certos objetivos préestabelecidos. Em situações em que a tomada de decisão está relacionada à alocação de recursos limitados, esta alocação é função da decisão e da racionalidade do agricultor que depende, por sua vez, de métodos eficientes que o auxiliem na otimização da sua decisão (Carvalho et al., 2000).

Em condições limitantes de água, técnicas de programação matemática, linear e não linear, têm sido empregadas como instrumentos para a alocação ótima de recursos em áreas irrigadas (Dantas Neto et al., 1997; Ghahraman \& Sepaskhah, 2002; Tsakiris \& Spiliotis, 2006; Borges Júnior et al., 2008a; Georgiou \& Papamichail, 2008) ou, ainda, no projeto de sistemas de irrigação (Lejano, 2006; Kale et al., 2008).

A programação linear é o método de programação matemática mais difundido para planejamento de empreendimentos agrícolas (Hardaker et al., 2004). Pode ser empregada, por exemplo, quando se tem o objetivo de maximizar o valor presente líquido do empreendimento, o que requer o conhecimento da taxa de desconto para aplicação em períodos de planejamento superiores a um ano. A associação da análise financeira com a gestão de recursos hídricos pode ser feita adotando-se restrições relativas à disponibilidade hídrica e se considerando, preferencialmente, a variabilidade sazonal.

$\mathrm{Na}$ análise financeira se comparam os benefícios e os custos em termos monetários, em uma base de tempo comum. Para esta finalidade pode-se utilizar indicadores, como a relação benefício-custo, o valor presente líquido e a taxa interna de retorno. $\mathrm{O}$ analista pode optar por um dos indicadores, dependendo da adequabilidade em relação à análise. Um projeto de irrigação é economicamente justificado quando os benefícios totais são maiores que os custos totais (Frizzone \& Andrade Júnior, 2005). Para isto, a relação benefício-custo deve ser maior que a unidade, o valor presente líquido positivo e a taxa interna de retorno maior que a taxa de desconto.

Além das análises de retorno financeiro, que podem ser executadas pelo emprego da programação linear, decisões acerca de investimentos são influenciadas por outros fatores relativos ao ambiente de implantação do projeto, dentre eles a impossibilidade de se prever as condições econômicas e ambientais que os envolvem. Desta forma, as decisões relativas a investimentos são tomadas considerando-se certo grau de incerteza e risco (Arêdes \& Pereira, 2008).

Com vistas ao planejamento, a utilidade de modelos determinísticos torna-se relativamente limitada em empreendimentos agrícolas devido às condições de risco sob as quais estes se desenvolvem. Isto implica na necessidade de não se desprezar, na maioria das vezes, a aleatoriedade de determinados coeficientes e de se introduzir o estudo do risco na análise do projeto (Silva Neto \& Stulp, 2000).

Técnicas de simulação de risco e análise de sensibilidade são complementares na análise de risco. Quando se aplica simulação de risco, o enfoque é na variável de saída, buscandose gerar, com base nas distribuições de probabilidade das variáveis e parâmetros de entrada e em correlações entre essas variáveis e parâmetros, a função de probabilidade acumulada para a variável de saída. Já na análise de sensibilidade o enfoque é sobre as variáveis e parâmetros de entrada, ou seja, verificase o impacto de variações em cada variável ou parâmetro de entrada isoladamente, sobre determinada variável de saída (Borges Júnior et al., 2008b).

É amplamente reconhecido o alto nível de incertezas impactantes especialmente sobre pequenas propriedades agrícolas em países em desenvolvimento (Fox et al., 2005). Na análise de risco aplicada a empreendimentos agrícolas, de agricultura irrigada ou de sequeiro, são inúmeros os fatores a serem considerados, relacionados diretamente ao processo produtivo ou a conjunturas econômicas.

Dentre os fatores relacionados ao processo produtivo, citamse as incertezas quanto à quantidade e frequência de chuvas, variabilidade na disponibilidade hídrica em fontes de abastecimento de sistemas de irrigação e a produtividade de culturas, de forma especial para cultivos de sequeiro. Incertezas quanto a custos de produção, preço de venda de produtos e taxas de juro, podem ser citadas como fatores de risco relacionados à economia.

O objetivo deste trabalho foi analisar padrões de cultivo, visando à otimização do uso dos recursos hídricos e a maximização do retorno financeiro para a agricultura familiar, num estudo de caso no Agreste Meridional Pernambucano, através da utilização da programação linear, além de avaliar o risco financeiro e quanto à escassez hídrica ao qual o empreendimento está exposto.

\section{Material E MÉTODOS}

Considerou-se uma propriedade localizada no município de Garanhuns, latitude $8^{\circ} 52^{\prime}$ Sul, longitude $36^{\circ} 28^{\prime}$ Oeste e altitude média de cerca de $820 \mathrm{~m}$, na porção alta da bacia hidrográfica do rio Mundaú e sul da mesorregião do Agreste Pernambucano. Procurou-se considerar uma propriedade de pequeno porte, com topografia e disponibilidade de recursos hídricos representativos da região. Os estudos de planejamento foram desenvolvidos com base apenas na área agricultável e em atividades agrícolas.

\section{Otimização do padrão de cultivo}

Para a otimização do padrão de cultivo foi utilizado um modelo de programação linear com horizonte de planejamento de três anos, implementado na planilha eletrônica Microsoft@ Excel e resolvido com uma ferramenta padrão, denominada Solver.

As culturas estudadas e épocas de cultivo são apresentadas na Tabela 1. Consideraram-se os cultivos de Feijão 2, Milho 2 e Mandioca como de sequeiro.

Dados para o estabelecimento dos coeficientes da função objetivo foram necessários para a construção da planilha, como: produtividade das culturas em diferentes manejos, requerimento de irrigação mensal das culturas e Irrigação Total Necessária (ITN) no ano de cultivo. Esses dados foram obtidos a partir de simulações realizadas com o Modelo Computacional 
Tabela 1. Época de plantio utilizada na simulação das culturas estudadas

\begin{tabular}{|c|c|c|c|c|c|c|c|c|c|c|c|c|}
\hline \multicolumn{13}{|c|}{ Cultivo* } \\
\hline \multicolumn{13}{|c|}{ Ano 1} \\
\hline Mês & Ban & Feij 1 & Feij 2 & Feij 3 & Feij 4 & Mil 1 & Mil 2 & Mand & Marac & Pim & Rep & Tom \\
\hline J aneiro & $x$ & $x$ & & & & $x$ & & & & & & \\
\hline Fevereiro & $X$ & $X$ & & & & X & & & & & & \\
\hline Março & $X$ & $X$ & $X$ & & $X$ & $X$ & & & $x$ & & & \\
\hline Abril & $x$ & & $x$ & & $x$ & $x$ & $x$ & $x$ & $x$ & & & \\
\hline Maio & $x$ & & $x$ & & $x$ & & $x$ & $x$ & $x$ & & & \\
\hline J unho & $x$ & & & & & & $x$ & $x$ & $x$ & & & \\
\hline J ulho & $x$ & & & & & & $x$ & $x$ & $x$ & & & \\
\hline Agosto & $x$ & & & & & & & $x$ & $x$ & & & \\
\hline Setembro & $x$ & & & & & & & $x$ & $x$ & & $x$ & $x$ \\
\hline Outubro & $x$ & & & $x$ & & & & $x$ & $x$ & $x$ & $x$ & $x$ \\
\hline Novembro & $x$ & & & $x$ & & & & $x$ & $x$ & $x$ & $x$ & $x$ \\
\hline Dezembro & $x$ & & & $x$ & & & & $x$ & $x$ & $x$ & $x$ & $x$ \\
\hline \multicolumn{13}{|c|}{ Ano 2} \\
\hline J aneiro & $x$ & $x$ & & & & $x$ & & $x$ & $x$ & $x$ & & \\
\hline Fevereiro & $X$ & $x$ & & & & $x$ & & $X$ & $X$ & $x$ & & \\
\hline Março & $x$ & $x$ & $x$ & & $X$ & $\mathrm{X}$ & & $x$ & $x$ & $x$ & & \\
\hline Abril & X & & $x$ & & $x$ & $x$ & $x$ & $x$ & $x$ & $x$ & & \\
\hline Maio & $x$ & & $x$ & & $x$ & & $x$ & $x$ & $x$ & & & \\
\hline J unho & $X$ & & & & & & $X$ & $x$ & $x$ & & & \\
\hline J ulho & $x$ & & & & & & $x$ & & $x$ & & & \\
\hline Agos to & X & & & & & & & & $X$ & & & \\
\hline Setembro & $x$ & & & & & & & & $x$ & & $x$ & $x$ \\
\hline Outubro & $x$ & & & $x$ & & & & & $x$ & $x$ & $x$ & $x$ \\
\hline Novembro & $x$ & & & $x$ & & & & & $x$ & $x$ & $x$ & $x$ \\
\hline Dezembro & $x$ & & & $x$ & & & & & $x$ & $x$ & $x$ & $x$ \\
\hline \multicolumn{13}{|c|}{ Ano 3} \\
\hline J aneiro & $x$ & $x$ & & & & $x$ & & & $x$ & $x$ & & \\
\hline Fevereiro & $x$ & $x$ & & & & $x$ & & & & $x$ & & \\
\hline Março & $X$ & $X$ & $X$ & & $X$ & $X$ & & & & $X$ & & \\
\hline Abril & $x$ & & $x$ & & $x$ & $x$ & $x$ & & & $x$ & & \\
\hline Maio & $x$ & & $x$ & & $x$ & & $x$ & & & & & \\
\hline J unho & $X$ & & & & & & $x$ & & & & & \\
\hline J ulho & $X$ & & & & & & $X$ & & & & & \\
\hline Agosto & $X$ & & & & & & & & & & & \\
\hline Setembro & $X$ & & & & & & & & & & $X$ & $X$ \\
\hline Outubro & $x$ & & & $x$ & & & & & & & $x$ & $x$ \\
\hline Novembro & $x$ & & & $x$ & & & & & & & $x$ & $x$ \\
\hline Dezembro & $X$ & & & $x$ & & & & & & & $x$ & $x$ \\
\hline
\end{tabular}

* Ban - Banana; Feij - Feijão; Mil. - Milho; Mand - Mandioca; Marac - Maracujá; Pim - Pimentão; Rep - Repolho; Tom - Tomate

para Suporte à Decisão em Irrigação e Drenagem - MCID (Ferreira et al., 2006; Borges Júnior et al., 2008c).

Os dados financeiros, como custo de produção e séries históricas de preços para o produtor, foram obtidos junto ao Banco do Brasil, na agência de Garanhuns. As séries históricas mensais de preços do período de janeiro de 2003 a janeiro de 2009, foram corrigidas segundo o IPCA (Índice de Preço ao Consumidor Amplo), para janeiro de 2009.

\section{Restrições do modelo de programação linear}

A restrição de terra foi definida estabelecendo-se uma divisão da fazenda em três subáreas (Área 1, Área 2 e Área 3) com 2 ha cada uma, para o plantio das culturas (Tabela 2).

O requerimento de irrigação das culturas foi utilizado para preencher a planilha referente às equações de restrições quanto à necessidade hídrica dos cultivos. A soma dos requerimentos hídricos deveria ser menor ou igual à disponibilidade mensal de água para irrigação (Tabela 2).
Tabela 2. Restrições quanto aos recursos para cada padrão de cultivo adotado no modelo de programação linear

\begin{tabular}{lcc}
\hline \multicolumn{1}{c}{ Restrição } & Unidade & Valor \\
Terra (em cada área) & (ha) & 2 \\
Mão-de-obra (mensal) & (dias-homem) & 125 \\
Água disponível para a irrigação (mensal) PC1* & $\left(\mathrm{m}^{3}\right)$ & 8.500 \\
Água disponível para a irrigação (mensal) PC2* & $\left(\mathrm{m}^{3}\right)$ & 4.000 \\
\hline * PC1 - padrão de cultivo 1: PC2 - padrão de cultivo 2 & &
\end{tabular}

As restrições referentes à necessidade de mão-de-obra para cada cultivo, em cada mês, foram determinadas a partir das planilhas de produção obtidas junto à agência bancária do Banco do Brasil, no município. A partir das discriminações dos componentes dos custos de produção, foi totalizada a quantidade de mão-de-obra necessária para os cultivos e esses valores foram distribuídos em base mensal, a depender dos procedimentos realizados em cada mês. A quantidade de mãode-obra que o agricultor tem disponível em sua propriedade está apresentada na Tabela 2. 
A disponibilidade mensal de água foi determinada com base em medidas do espelho d'água do reservatório, vazão afluente ao reservatório e quantidade de água que se deve deixar livre para os produtores, a jusante. Foram considerados dois padrões de cultivo relacionados a diferentes valores de disponibilidade hídrica: o primeiro padrão de cultivo, $\mathrm{PC} 1$, com $8.500 \mathrm{~m}^{3} \mathrm{de}$ disponibilidade hídrica mensal e o segundo padrão de cultivo, PC2, com disponibilidade mensal de $4.000 \mathrm{~m}^{3}$ (Tabela 2).

Nas restrições de produção (Tabela 3 ) foram considerados valores hipotéticos, seguindo-se os critérios: (a) diversificar os produtos na propriedade estabelecendo, assim, uma produção máxima para algumas culturas; (b) simular necessidades de produção mínima para atender a contratos pré-estabelecidos e/ou para outras atividades na propriedade, como, por exemplo, a produção de ração animal ou farinha; (c) representar as capacidades de armazenamento ou processamento e capacidade de mercado.

Tabela 3. Restrições quanto à produção adotadas no modelo de programação linear

\begin{tabular}{lccc}
\hline \multicolumn{1}{c}{ Cultura } & Unidade & Mínima & Máxima \\
Banana & t ano $^{-1}$ & 10 & 20 \\
Feijão & t safra $^{-1}$ & $*$ & 3 \\
Mandioca & t safra $^{-1}$ & 5 & 10 \\
Maracujá & t ano $^{-1}$ & 10 & 30 \\
Milho & t safra $^{-1}$ & 1 & 5 \\
Pimentão & t safra $^{-1}$ & $*$ & 70 \\
Repolho & t safra $^{-1}$ & $*$ & 50 \\
Tomate & t safra $^{-1}$ & $*$ & 100 \\
\hline T Sem restriça mín
\end{tabular}

* Sem restrição mínima de produção

\section{Funçãoobjetivo}

O critério de avaliação econômica de projetos utilizado no estudo foi o valor presente líquido total, VPLt, que representa o lucro obtido pelo produtor com as atividades que ele trabalhará, ou seja, as culturas que ele irá implantar, corrigidos para o tempo presente, segundo uma taxa de desconto. Desta maneira, o modelo de programação linear visa à maximização do valor presente líquido total da propriedade. A função objetivo do modelo é:

$$
\text { Maximizar VPLt }=\mathrm{L}_{0}+\sum_{\mathrm{t}=1}^{\mathrm{n}} \frac{\mathrm{L}_{\mathrm{t}}}{(1+\mathrm{i})^{\mathrm{t}-0,5}}
$$

em que:

VPLt - valor presente líquido total, $R \$$

$\mathrm{L}_{0}$ - custos no tempo $\mathrm{t}=0$

$\mathrm{L}_{\mathrm{t}}$ - valor do fluxo líquido do projeto no ano $t, \mathrm{R} \$$

$\mathrm{n}$ - horizonte do projeto, igual a três anos

t - tempo, anos

i - taxa de desconto, decimal.

Os coeficientes da função objetivo foram determinados com os seguintes dados:

- Custo de produção, exceto o de irrigação;

- Custo operacional da irrigação: foi considerado o custo de energia necessária para fornecer o valor total de ITN aos cultivos, mas não foi considerada a cobrança pelo uso da água, ainda não implementada na região.

- Preço de venda: os preços de venda dos diversos produtos foram obtidos junto ao Banco do Brasil.

- Produtividade: foram obtidas as produtividades em diferentes tipos de manejo de irrigação das culturas ou cultivo de sequeiro, através do emprego no modelo de simulação MCID.

- Taxa de desconto anual: foi considerada a taxa de desconto de $8 \%$ ao ano; este valor foi obtido pela diferença arredondada entre a taxa Selic anual, para junho de 2007, igual a 12,43\% e a meta de inflação de 2007 do Conselho Monetário Nacional, igual a 4,50\% ao ano.

\section{Análise de sensibilidade}

A análise de sensibilidade da solução do modelo de programação linear foi realizada a partir do Relatório de Sensibilidade, gerado pela aplicação do Solver. Esta análise consistiu na avaliação da estabilidade da solução encontrada com o modelo de programação linear, por meio de informações como custos reduzidos e preços sombra (Borges Júnior et al., 2008a).

\section{Análise de risco}

$\mathrm{Na}$ análise de risco visou-se obter a função de probabilidade acumulada das variáveis de saída VPLt e requerimentos de irrigação, com base nas distribuições de probabilidade e correlações de variáveis de entrada (Borges Junior et al., 2008a). A técnica de amostragem para a simulação utilizada foi o método Monte Carlo, que é um tipo de simulação usada em modelos envolvendo eventos probabilísticos.

Para processamento dessas simulações foi empregado o programa computacional @RISK (Hardaker et al., 2004; Palisade Corporation, 2009), implementado junto à planilha eletrônica Excel, em que as variáveis de entrada foram consideradas de forma estocástica. Esta análise foi aplicada sobre a estrutura do modelo de programação linear. Na simulação, foram feitas 10.000 iterações com o programa.

As funções de distribuição de probabilidade das variáveis de entrada foram:

- Preço: função de distribuição triangular, com valores mínimo, mais provável e máximo iguais a 50, 100 e 120\%, respectivamente, do valor de preço de venda empregado no modelo de programação linear.

- Produtividade: função de distribuição da probabilidade normal, com desvio padrão determinado de acordo com os valores de coeficiente de variação e média, obtidos da modelagem executada com o MCID. Para culturas irrigadas, o coeficiente de variação foi 0,03 , devido ao baixo desvio padrão verificado para esses cultivos, como efeito da mitigação do risco, proporcionada pela irrigação. Para cultivos de sequeiro os coeficientes de variação (Tabela 4) foram determinados a partir da média de produtividade e do desvio padrão encontrados nas simulações com o MCID.

- Taxa de desconto anual: função de distribuição triangular com valor mínimo de 7\%, valor mais provável de $8 \%$ e valor máximo de $10 \%$. 
Tabela 4. Coeficientes de variação dos cultivos de sequeiro

\begin{tabular}{cccc}
\hline Cultura & \multicolumn{3}{c}{ Coeficiente de variação } \\
& Feijão $^{1}$ & Milho $^{2}$ & Mandioca \\
Área 1 & 0,379 & 0,948 & 0,425 \\
Área 2 & 0,374 & 1,091 & 0,452 \\
Área 3 & 0,363 & 0,950 & 0,411 \\
\hline
\end{tabular}

1 - Plantio em março; 2 - Plantio em abril

- Custo de produção sem irrigação: função de distribuição triangular, com valor mínimo, valor mais provável e valor máximo iguais a 90, 100 e 120\%, respectivamente, do valor do custo de produção empregado no modelo de programação linear.

- Requerimento mensal de irrigação: função de distribuição normal padrão com truncamento nos extremos, em níveis de probabilidade de 5 e $95 \%$. O truncamento visa evitar valores extremamente altos ou baixos de determinada variável, gerados aleatoriamente no método Monte Carlo.

Segundo Borges Júnior et al. (2008b), considera-se que o coeficiente de correlação dos requerimentos mensais de irrigação é igual a um. Em cada mês se obtêm, aplicando-se o método de Monte Carlo, um valor para a variável normal padrão e só então se calculam os valores mensais para o requerimento de irrigação, por meio da equação:

$$
\mathrm{wr}_{\mathrm{jym}}=\mathrm{DP} \mathrm{w}_{\mathrm{w}} \cdot \mathrm{Z}+\mathrm{w}_{\mathrm{jym}}
$$

em que:

$\mathrm{wr}_{\mathrm{jym}}$ - valor realizado para $\mathrm{w}_{\mathrm{jym}}$ equivalente ao valor realizado de $\mathrm{Z}, \mathrm{m}^{3} \mathrm{ha}^{-1}$

DPw - desvio padrão de $\mathrm{w}_{\mathrm{jym}}, \mathrm{m}^{3} \mathrm{ha}^{-1}$

Z - variável normal padrão.

$\mathrm{w}_{\mathrm{jym}}$-requerimento hídrico para a atividade (cultivo) $\mathrm{j}$, ano y e mês $m, m^{3} h a^{-1}$.

As funções de distribuição de probabilidade para as variáveis de entrada preço, taxa de desconto e custo, foram definidas com o objetivo de tornar o modelo mais conservador. As funções triangulares empregadas são assimétricas, no sentido de acarretar menores valores presentes líquidos totais do que se fossem simétricas, pois existe maior probabilidade de haver maiores valores de taxa de desconto, menores valores do preço de venda dos produtos e maiores valores de custo.

\section{RESULTADOS E DISCUSSÃO}

\section{Otimização do valor presente líquido total}

O modelo de programação linear proposto resultou em dois padrões de cultivo, apresentados nas Tabelas 5 e 6 , considerando-se as restrições de água, mão-de-obra e de terra e satisfazendo as exigências de áreas mínimas e máximas para atendimento das restrições de produção relativas a algumas atividades.

No primeiro padrão de cultivo (PC1), com restrição hídrica mensal para irrigação de $8.500 \mathrm{~m}^{3}$, o valor presente líquido total, VPLt (R\$), apontado como ótimo, foi de R \$ 157.879,73, o que representa o lucro corrigido pela taxa de desconto com período
Tabela 5. Padrão de Cultivo 1 (PC1): solução ótima encontrada pelo modelo de programação linear para um volume mensal de água igual a $8.500 \mathrm{~m}^{3}$

\begin{tabular}{lccc}
\hline \multicolumn{1}{c}{ Cultivo } & $\begin{array}{c}\text { Ano 1 } \\
\text { (ha) }\end{array}$ & $\begin{array}{c}\text { Ano 2 } \\
\text { (ha) }\end{array}$ & $\begin{array}{c}\text { Ano 3 } \\
\text { (ha) }\end{array}$ \\
Banana 1 área* 1 & 0,42 & 0,42 & 0,42 \\
Banana área 2 & 0,15 & 0,15 & 0,15 \\
Maracujá área 2 & 1,85 & 1,85 & 1,85 \\
Mandioca área 3 & 0,67 & 0,67 & -- \\
Feijão 1 área 3 & 1,33 & 0,67 & 0,57 \\
Feijão 3 área 3 & 0,95 & 0,96 & 2,00 \\
Feijão 4 área 1 & 1,56 & -- & -- \\
Feijão 4 área 2 & -- & -- & 1,85 \\
Feijão 4 área 3 & 0,42 & 0,03 & 0,15 \\
Milho 1 área 3 & 0,25 & 0,25 & 0,25 \\
Pimentão área 1 & 1,58 & 1,58 & -- \\
Pimentão área 3 & 0,38 & 0,38 & -- \\
Tomate área 1 & -- & -- & 1,08 \\
\hline
\end{tabular}

* No modelo a área total da propriedade foi dividida em três

Tabela 6. Padrão de Cultivo 2 (PC2): solução ótima encontrada pelo modelo de programação linear para um volume mensal de água igual a $4.000 \mathrm{~m}^{3}$

\begin{tabular}{lccc}
\hline \multicolumn{1}{c}{ Cultivo } & $\begin{array}{c}\text { Ano 1 } \\
\text { (ha) }\end{array}$ & $\begin{array}{c}\text { Ano 2 } \\
\text { (ha) }\end{array}$ & $\begin{array}{c}\text { Ano 3 } \\
\text { (ha) }\end{array}$ \\
Banana 1 área* 1 & 0,57 & 0,57 & 0,57 \\
Maracujá área 2 & 0,82 & 0,82 & 0,82 \\
Mandioca área 1 & -- & 0,68 & -- \\
Mandioca área 3 & 0,67 & -- & -- \\
Feijão 1 área 3 & -- & 0,68 & 0,27 \\
Feijão 3 área 3 & 1,64 & 0,08 & 1,20 \\
Feijão 4 área 1 & 0,47 & 0,75 & 0,47 \\
Feijão 4 área 2 & 1,17 & -- & 1,53 \\
Feijão 4 área 3 & 0,36 & -- & -- \\
Milho 1 área 1 & 0,25 & -- & -- \\
Milho 1 área 2 & -- & 0,16 & 0,25 \\
Milho 1 área 3 & -- & 0,08 & -- \\
Pimentão área 2 & 1,01 & 0,22 & -- \\
Pimentão área 3 & 1,24 & -- & -- \\
Tomate área 1 & -- & -- & 1,08 \\
\hline * No modelo a áre total propriedade foi dividida om três & &
\end{tabular}

* No modelo a área total da propriedade foi dividida em três

de planejamento de três anos e área total disponível de 6 ha, ou seja, o retorno financeiro foi de $\mathrm{R} \$ 8.771,10$ (ha ano $)^{-1}$.

Para o segundo padrão de cultivo (PC2), com restrição hídrica mensal para irrigação de $4.000 \mathrm{~m}^{3}$, o valor presente líquido apontado como ótimo foi de $\mathrm{R} \$ 148.117,90$, representando $\mathrm{R} \$$ $8.228,77$ (ha ano $^{-1}$, considerando-se o mesmo período de planejamento e dimensão da área.

O percentual de ocupação da área agricultável para os três anos de planejamento no Padrão de Cultivo 1 (PC1) foi de 69\%; no Padrão de Cultivo 2 (PC2), foi de $60 \%$. A ocupação da área não foi total (100\%) no PC1 devido, principalmente, à restrição de mão-de-obra limitante em alguns meses do planejamento. Já no PC2, as restrições de disponibilidade hídrica e mão-de-obra foram significantes na taxa de ocupação do terreno. Adicionalmente ao efeito das restrições, em ambos os padrões de cultivo existiram alguns meses em que o terreno foi deixado livre para posterior estabelecimento de cultivos mais rentáveis economicamente.

Andrade et al. (2008) utilizaram a programação linear para estabelecer um padrão de cultivo ótimo visando à maximização 
do valor presente líquido total em uma propriedade do perímetro irrigado de Gorutuba, MG, com restrição de terra de 200 ha, de mão-de-obra de 6.000 dias homens, disponibilidade mensal de água para a irrigação de $155.000 \mathrm{~m}^{3}$ e horizonte de planejamento de quatro anos. O padrão de cultivo apontado como ótimo, em termos de maximização do valor presente líquido total, foi de $\mathrm{R} \$ 2.918 .744,05$, representando $\mathrm{R} \$ 3.648,43$ (ha ano) ${ }^{-1}$, com base em dados financeiros atualizados para dezembro de 2006. O valor corrigido para janeiro de 2009, com base no IPCA, é de R\$ 4.055,37 (ha ano $)^{-1}$, cerca da metade do encontrado neste trabalho o que, provavelmente, é decorrente de restrições mais severas em termos de mão-de-obra e produção e devido, também, a um efeito mais expressivo da restrição hídrica frente a uma demanda maior de água para irrigação.

Dantas Neto et al. (1997), utilizando modelo de programação linear para estimar a maximização dos lucros da área de colonização do projeto Senador Nilo Coelho em Petrolina, PE, com área total de 7.424 ha, verificaram que o retorno financeiro foi de US\$ 22.634.044,00. Considerando a cotação do dólar para dezembro de 2008, verificou-se que o retorno financeiro foi de R \$ 52.510.982,08, totalizando R \$ 7.073, 14 (ha. ano) ${ }^{-1}$, utilizando-se o seguinte padrão de cultivo: feijão Phaseolus (714 ha), melancia (714 ha), pimentão (714 ha), tomate (4.281 ha), cebola (357 ha) e banana ( 818 ha). As restrições atuantes no modelo, foram: volume de água mensal, volume de água anual, terra e mercado, sendo que a restrição atuante no modelo foi a disponibilidade anual de água.

Na Figura 1 apresenta-se a linha de soluções da programação linear no plano do valor presente líquido total (VPLt) e restrição mensal de água para a irrigação no Padrão de Cultivo 2. Observase que, para valores de restrição mensal maiores que $4.000 \mathrm{~m}^{3}$, a curva passa a ter padrão assintótico, com pequena variação no VPLt até o valor máximo de R \$ 148.117,90.

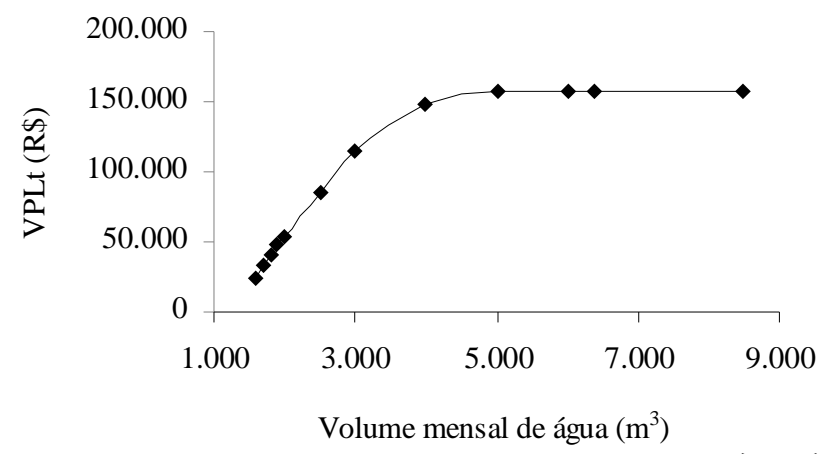

Figura 1. Variação do valor presente líquido total (VPLt) com relação à restrição mensal de água para irrigação no Padrão de Cultivo 2

Analisando a disponibilidade mensal de água nesta propriedade verifica-se, para volumes inferiores a $1.500 \mathrm{~m}^{3}$, que a solução ótima do modelo de programação linear não foi encontrada em razão da impossibilidade de satisfação conjunta das restrições do modelo, sobretudo daquelas que limitam a produtividade mínima de algumas culturas.

Na Tabela 7 percebe-se que a diferença no VPLt entre os dois padrões de cultivo é de $6,2 \%$, enquanto a diferença na disponibilidade de água para a irrigação é cerca de 52,9\% e o requerimento hídrico total, para os três anos, nos meses de pico (janeiro, fevereiro, março, outubro, novembro, dezembro) foi de $24,1 \%$.

Tabela 7. Comparação entre o valor presente líquido total (VPLt), disponibilidade de água para irrigação e requerimento hídrico total (para os três anos) nos meses de pico, para os dois padrões de cultivo

\begin{tabular}{lccc}
\hline Padrão de cultivo & $\begin{array}{c}\text { VPLt } \\
\text { (R\$) }\end{array}$ & $\begin{array}{c}\text { Restrição } \\
\text { mensal }\left(\mathbf{m}^{3}\right)\end{array}$ & $\begin{array}{c}\text { Requerimento total nos } \\
\text { meses de pico }\left(\mathbf{m}^{3}\right)\end{array}$ \\
\hline Padrão de cultivo 1 & $157.879,73$ & 8.500 & 94.759 \\
Padrão de cultivo 2 & $148.117,93$ & 4.000 & 71.886 \\
Diferença & $6,2 \%$ & $52,9 \%$ & $24,1 \%$ \\
\hline Valores obtidos a partir da diferenca nos valores encontrados nos dois cultivos edepois comparados
\end{tabular}

Valores obtidos a partir da diferença nos valores encontrados nos dois cultivos e depois comparados com o Padrão de Cultivo 1

Esses dados evidenciam que, através do planejamento de alocação de área e época para os cultivos, pode-se estabelecer padrões de cultivo que proporcionem um retorno financeiro próximo ao ótimo com expressiva economia de recursos hídricos, o que se configura num importante aspecto para a região de estudo, com baixa relação entre disponibilidade e demanda hídrica, conforme dados da ANA (2009).

\section{Análise de sensibilidade}

Com base nos valores de custos reduzidos, os quais indicam quanto o valor presente líquido total (função objetivo) diminuiria se uma unidade da correspondente atividade, excluída da solução ótima, fosse considerada no padrão de cultivo (Borges Júnior et al., 2008a), constatou-se que as atividades que proporcionariam maior redução do retorno financeiro nos dois padrões de cultivo, caso empreendidas, estão relacionadas às culturas de repolho e tomate. A atividade que provocaria a maior diminuição do lucro é o tomate implantado na Área 2, no Ano 1 de planejamento nos dois padrões de cultivo.

No Padrão de Cultivo 1 foram atuantes as restrições de terra, mão-de-obra e produção (Tabelas 2 e 3), sendo que a principal restrição atuante no modelo de programação linear foi a disponibilidade de terra para o plantio das culturas (Tabela 8).

Para restrições do tipo menor ou igual, o preço sombra representa o quanto o valor presente líquido total aumentaria com o acréscimo de uma unidade adicional na restrição correspondente: o contrário ocorre para restrições do tipo maior ou igual.

O maior valor de preço sombra para a restrição de terra foi encontrado no mês de março, no segundo ano, para a segunda área; o aumento de 1 ha resultaria em um incremento de $\mathrm{R} \$$ 698,38 no retorno financeiro do empreendimento.

A restrição de mão-de-obra foi atuante no mês de outubro, nos anos 1 e 2, devido ao estabelecimento dos cultivos de Banana, Maracujá, Mandioca, Feijão 3, Pimentão e Tomate, e no mês de setembro, Ano 3, em virtude dos altos requerimentos para os cultivos de Banana e Tomate. O aumento de um diahomem na restrição de mão-de-obra no mês de outubro, Ano 1, resultaria em um incremento expressivo de $\mathrm{R} \$ 560,25$ no valor no retorno financeiro do empreendimento. 
Tabela 8. Restrições atuantes no modelo de programação linear com maximização do valor presente líquido total para o Padrão de Cultivo 1, preços sombra, acréscimos e decréscimos permissíveis

\begin{tabular}{lccrc}
\hline Tipo de restrição & Nome e unidade da restrição & Preço sombra & Acréscimo permissível & Decréscimo permissível \\
Água & $\mathrm{m}^{3}$ & -- & -- & - \\
Mão-de obra & Out ano 1 (dias homem) & 560,25 & 1,34 & 17,23 \\
Mão-de obra & Out ano 2 (dias homem) & 521,34 & 17,83 & 16,80 \\
Mão-de obra & Set ano 3 (dias homem) & 105,79 & 2,73 & 103,36 \\
Terra & Mar ano 3 área 1 (ha) & 491,49 & 0,15 & 0,00 \\
Terra & Abr ano 3 área 1 (ha) & 39,80 & 0,00 & 0,56 \\
Terra & Mar ano 1 área 2 (ha) & 616,18 & 0,00 & 0,00 \\
Terra & Dez ano 1 área 2 (ha) & 428,23 & 0,00 & 0,00 \\
Terra & Mar ano 2 área 2 (ha) & 698,38 & 0,00 & 0,00 \\
Terra & Dez ano 2 área 2 (ha) & 367,70 & 0,00 & 0,00 \\
Terra & Jan ano 3 área 2 (ha) & 28,59 & 0,00 & 0,00 \\
Terra & Mar ano 3 área 2 (ha) & 482,16 & 0,15 & 0,15 \\
Terra & Abr ano 3 área 2 (ha) & 46,12 & 0,00 & 6,86 \\
Produção & Maracujá (t ha) & 453,56 & 0,66 & 3,00 \\
Produção & Feijão 3 ano 2 & 316,05 & 0,00 & 0,00 \\
Produção & Milho 2 ano 3 & $-690,44$ & 0,51 & 2,07 \\
Produção & Banana ano 2 & $-1,901,91$ & 0,79 & 5,00 \\
Produção & Mandioca ano 2 & $-251,24$ & 0,22 & 0,00 \\
Produção & Milho 2 ano 1 & $-805,34$ & 0,59 & 1,00 \\
Produção & Milho ano 1 & $-61,45$ & 2,62 & 1,00 \\
Produção & Milho ano 3 & 2,25 & \\
\hline
\end{tabular}

De acordo com Ponciano et al. (2004), a importância do custo da mão-de-obra na determinação do resultado financeiro dos sistemas agrícolas é reflexo direto da grande exigência desse fator de produção ao longo do ciclo produtivo das culturas. Em vista disto, além dos aspectos econômicos analisados o fato da agricultura ser uma atividade com alta demanda em mão-de-obra, implica em que ela pode gerar, também, grandes benefícios sociais, mediante a criação de empregos.

Conforme Borges Júnior (2004), acréscimos e decréscimos permissíveis indicam o intervalo no qual o valor da restrição pode ser variado, sem que haja alteração no valor do preço sombra. Quando o valor da restrição limitante é alterado neste intervalo, os níveis das atividades serão alterados mas não será alterada a base da solução ótima, ou seja, não será alterado o conjunto de atividades presentes na solução ótima.

Com relação à produção, caso a restrição aumentasse em uma tonelada para a cultura de maracujá, isto seria refletido num aumento de $\mathrm{R} \$ 453,56$ no valor presente líquido total. O valor do preço sombra não seria alterado no intervalo entre 23,14 (diferença entre o valor da restrição e o decréscimo permissível) a 30,66 (soma entre o valor da restrição e o acréscimo permissível) toneladas de maracujá (Tabela 8).

Para as culturas de banana, mandioca e milho foram encontrados valores negativos de preço sombra. Valores negativos ocorrem quando uma condição de restrição de valor mínimo é limitante e reflete o decréscimo no retorno financeiro para cada tonelada de incremento no valor da restrição mínima para a produção da cultura correspondente. Por exemplo, o aumento de uma tonelada na restrição de produção mínima de Milho 2 no primeiro ano acarretaria um decréscimo de $\mathrm{R} \$ 805,34$ no valor presente líquido total.

Observa-se o expressivo impacto do ambiente de produção sobre o planejamento. Andrade et al. (2008), procurando estabelecer um padrão ótimo de cultivo em uma propriedade do perímetro irrigado de Gorutuba, MG, verificaram que as restrições relativas à terra não foram atuantes, em razão do efeito mais expressivo das restrições de água e mão-de-obra, diferentemente do que se verificou no estudo aqui apresentado.

No Padrão de Cultivo 2 observa-se, na Tabela 9, que restrições de todos os tipos (água, terra, mão-de-obra e produção) foram limitantes.

O maior preço sombra encontrado para a restrição hídrica foi no mês de dezembro, Ano 2, indicando que cada unidade de $\mathrm{m}^{3}$ disponibilizado para a irrigação neste mês resultaria em um aumento de $\mathrm{R} \$ 8,00$ no retorno financeiro da propriedade, no intervalo entre 3.998,56 (diferença entre o valor da restrição e o decréscimo permissível) a 4.146,58 $\mathrm{m}^{3}$ (soma entre o valor da restrição e o acréscimo permissível) para esta restrição.

A restrição de mão-de-obra foi limitante nos mesmos meses do Padrão de Cultivo 1, com diferentes valores de preço sombra e acréscimos e decréscimos permissíveis.

A restrição de disponibilidade de terra foi menos atuante (número de casos) neste padrão de cultivo, comparativamente ao Padrão de Cultivo 1. O maior preço sombra encontrado para a disponibilidade de área foi para o mês de março Ano 2, Área 3 , cujo aumento de um hectare resultaria na adição de $R$ \$ 706,94 ao valor presente líquido total.

Quanto à produção, a restrição máxima foi atuante apenas no cultivo de feijão. Por outro lado, restrições de produção mínima foram atuantes resultando em preços sombra negativos para as culturas de banana, mandioca e milho.

\section{Análise de risco}

Para a variável de saída valor presente líquido total (VPLt), no Padrão de Cultivo 1, a média de R \$130.086,00, resultante das simulações, foi consideravelmente inferior $(17,60 \%)$ ao valor máximo encontrado no modelo de programação linear, igual a R \$ 157.879,00; para o Padrão de Cultivo 2, houve redução de $17,46 \%$ no retorno financeiro, enquanto no modelo de programação linear se obteve o VPLt de R $\$ 148.117,90$, a simulação de risco indicou uma média de $\mathrm{R} \$ 122.252,80$. 
Tabela 9. Restrições atuantes no modelo de programação linear com maximização do valor presente líquido total para 0 Padrão de Cultivo 2, preços sombra, acréscimos e decréscimos permissíveis

\begin{tabular}{lccrc}
\hline \multicolumn{1}{c}{ Tipo de restrição } & Nome e unidade da restrição & Preço sombra & Acréscimo permissível & Decréscimo permissível \\
Água & Mar ano $1\left(\mathrm{~m}^{3}\right)$ & 1,00 & 349,742 & 460,09 \\
Água & Dez ano 2 (m $\left.\mathrm{m}^{3}\right)$ & 8,0 & 146,58 & 1,44 \\
Mão-de obra & Out ano 1 (dias homem) & 562,16 & 3,62 & 0,05 \\
Mão-de obra & Out ano 2 (dias homem) & 356,96 & 0,06 & 6,49 \\
Mão-de obra & Set ano 3 (dias homem) & 101,81 & 22,75 & 05,61 \\
Terra & Mar ano 3 área 2 (ha) & 510,75 & 0,52 & 0,000 \\
Terra & Abr ano 3 área 2 (ha) & 1,60 & 0,000 & 0,27 \\
Terra & Mar ano 1 área 3 (ha) & 30,96 & 0,49 & 0,37 \\
Terra & Dez ano 1 área 3 (ha) & 1,35 & 0,000 & 0,17 \\
Terra & Fev ano 2 área 3 (ha) & 13,94 & 0,000 & 0,08 \\
Terra & Mar ano 2 área 3 (ha) & 706,94 & 0,000 & 0,000 \\
Produção & Feijão 4 ano 3 & 89,53 & 0,41 & 0,71 \\
Produção & Milho 2 ano 3 & $-677,50$ & 0,25 & 0,000 \\
Produção & Banana ano 2 & $-2237,15$ & 0,019 & 2,56 \\
Produção & Mandioca ano3 & $-153,77$ & 1,64 & 0,33 \\
Produção & Milho 2 ano 2 & $-710,24$ & 0,000 & 0,000 \\
Produção & Milho ano 2 & $-92,34$ & 0,47 & 0,004 \\
\hline
\end{tabular}

A instabilidade quanto ao retorno financeiro é consequência da variabilidade nos preços de produtos agrícolas, custos, produtividades e fatores climáticos, dentre outros, sendo representada na análise de risco através das distribuições de probabilidade das variáveis de entrada. A obtenção de menores valores médios do valor presente líquido total com a simulação de risco, em comparação com os obtidos com a programação linear, decorre da assimetria das distribuições de probabilidade das variáveis de entrada: preços dos produtos, custo de produção, exceto irrigação e taxa de desconto anual, em concordância com a observação de Andrade et al. (2008).

Na Figura 2, em que são apresentadas curvas de distribuição de probabilidade acumulada para a variável de saída valor presente líquido total (VPLt), todos os valores resultantes das simulações foram positivos, evidenciando que, nos dois padrões de cultivo, o empresário rural não está frente ao risco de fracasso financeiro (VPLt $<0$ ), considerando-se a base de dados e premissas empregadas na análise.

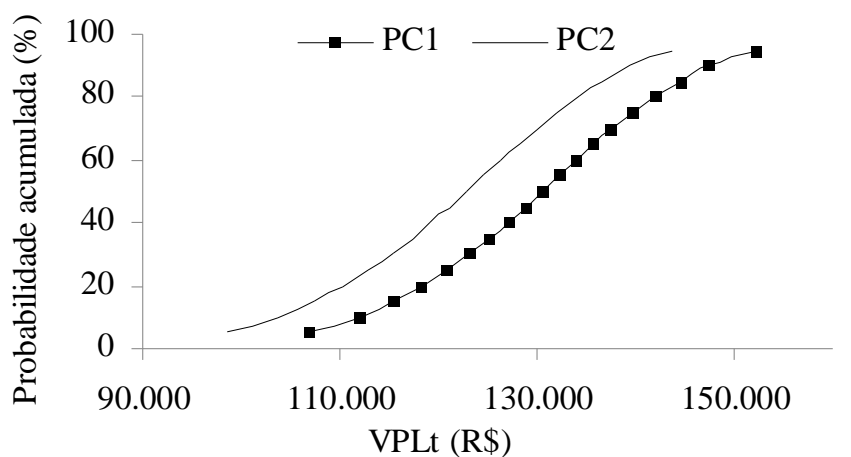

Figura 2. Curvas de distribuição de probabilidade acumulada do valor presente líquido total (VPLt) para os padrões de cultivo 1 (PC1) e 2 (PC2)

Segundo Arêdes \& Pereira (2008), a condição necessária para um empreendimento agrícola ser economicamente viável é que o indicador econômico valor presente líquido gerado seja positivo durante o período de planejamento. Desta forma, analisando-se a Figura 2 a partir da distribuição acumulada de probabilidade do VPLt, verifica-se a inexistência de risco financeiro para ambos os padrões de cultivo.

Na Figura 3 percebe-se grande discrepância com relação aos valores de Irrigação Total Necessária, ITN, no ano de maior requerimento hídrico (Ano 2), nos dois padrões de cultivo. São apresentadas curvas de distribuição de probabilidade acumulada para a variável de saída ITN, com base em simulações com o @ Risk, baseadas no método de Monte Carlo. Percebe-se que, para o Padrão de Cultivo 2, o requerimento de irrigação é consideravelmente menor que o verificado no Padrão de Cultivo 1. Por exemplo, considerando a probabilidade acumulada de $40 \%$, a ITN foi aproximadamente igual a 25.000 $\mathrm{m}^{3}$ no Padrão de Cultivo 2 e $35.000 \mathrm{~m}^{3}$ no Padrão de Cultivo 1.

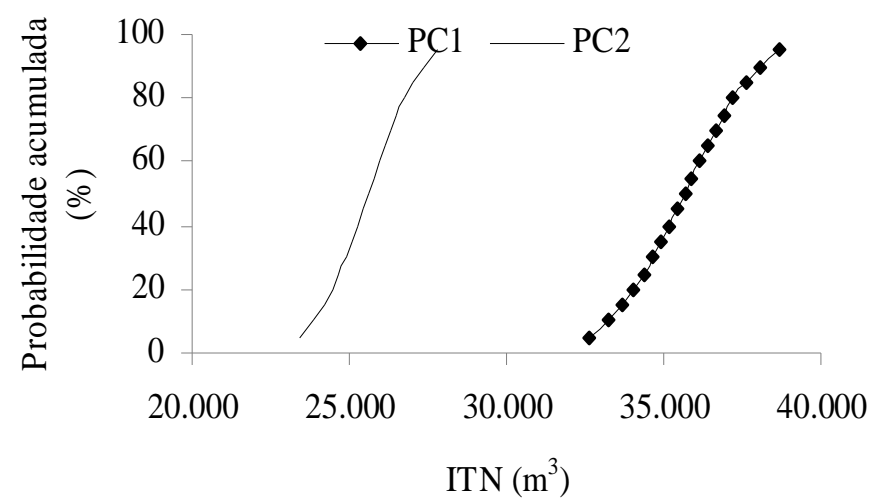

Figura 3. Curvas de distribuição de probabilidade acumulada da irrigação total necessária, ITN , $\mathrm{m}^{3}$, para os padrões de cultivo 1 (PC1) e 2 (PC2), no ano de maior requerimento hídrico (Ano 2)

A Figura 4 representa, graficamente, o requerimento de irrigação total (RIT) no mês de dezembro do Ano 2 (mês de máximo RIT). Nota-se que a quantidade de água necessária para a irrigação no Padrão de Cultivo 2 (PC2) é consideravelmente menor que no Padrão de Cultivo 1 (PC1) e que a variabilidade do RIT no PC1 é maior que no PC2, o que se constata por meio da menor inclinação da curva de probabilidade acumulada. 


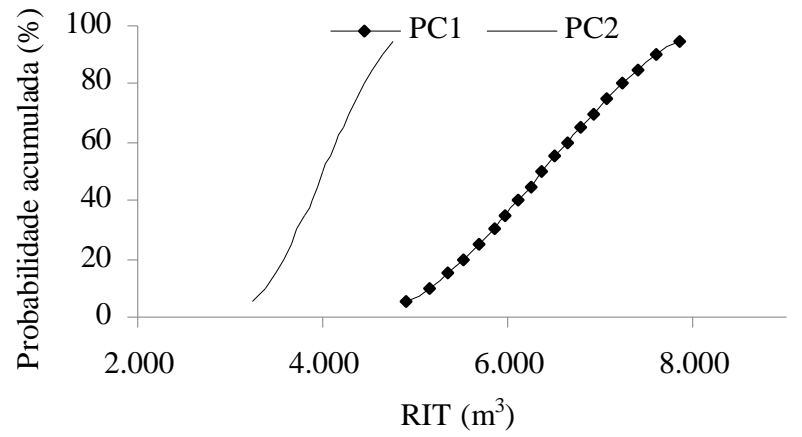

Figura 4. Requerimento de irrigação total (RIT) no mês de dezembro, Ano 2, nos padrões de cultivo 1 (PC1) e 2 (PC2)

\section{Conclusões}

1. Obtiveram-se planos ótimos de cultivo para os dois padrões de demanda hídrica utilizados nos estudos, contemplando os cultivos de banana, maracujá, mandioca, feijão, milho, pimentão e tomate.

2. Na análise de risco observou-se que os valores encontrados de valor presente líquido total (VPLt), no período de planejamento de três anos, resultantes das simulações, foram positivos nos dois padrões de disponibilidade hídrica, evidenciando viabilidade financeira para qualquer um dos padrões de cultivo.

3. Por meio da análise de risco pode-se perceber que houve redução do valor presente líquido total em $17,60 \%$ no Padrão de Cultivo 1 e em 17,46\% no Padrão de Cultivo 2, em relação aos valores resultantes do modelo de programação linear.

4. Comparando os dois padrões de cultivo, percebe-se a possibilidade de expressiva economia dos recursos hídricos, apesar de uma pequena redução do retorno financeiro do empreendimento, o que indica a aplicabilidade da metodologia apresentada para a gestão dos recursos hídricos, especialmente em regiões em que a relação entre disponibilidade e demanda hídrica é crítica, como no Agreste Meridional Pernambucano.

\section{LITERATURA CITADA}

ANA - Agência Nacional de Águas. Conjuntura dos recursos hídricos no Brasil 2009. Brasília: ANA, 2009. 204p.

Andrade, C. L. T.; Borges Júnior, J. C. F.; Lima, R. B. M.; Oliveira, F. G.; Ferreira, P. A.; Egídio, M. A. R. Avaliação de estratégias de produção em agricultura irrigada no perímetro de irrigação do Gorutuba. Embrapa Milho e Sorgo (Doc. 73), 2008. 151p. http://www.cnpms.embrapa.br. 15 Abr. 2010.

Arêdes, A. F.; Pereira, M. W. G. Análise econômica da produção de café arábica: um estudo de caso com simulações de Monte Carlo para sistemas de baixa e alta produtividade. Informações Econômicas, v.38, n.4, p.19-30- 2008.

Borges Júnior, J. C. F. Modelo computacional para a tomada de decisão em agricultura irrigada. Viçosa: UFV, 2004. 250p. Tese Doutorado

Borges Júnior, J. C. F.; Ferreira, P. A.; Andrade, C. L. T.; HeddenDunkhorst, B. Computational modeling for irrigated agriculture planning. Part I: General description and linear programming. Engenharia Agrícola, v.28, n.3, p.471-482, 2008a.
Borges Júnior, J. C. F.; Ferreira. P. A.; Andrade, C. L. T.; HeddenDunkhorst, B.; Computational modeling for irrigated agriculture planning. Part II: Risk analysis. Engenharia Agricola, v.28, n.3, p.483-493, 2008b.

Borges Júnior, J. C. F.; Ferreira, P. A.; Hedden-Dunkhorst, B.; Andrade, C. L. T. Modelo computacional para suporte à decisão em áreas irrigadas. Parte I: Desenvolvimento e análise de sensibilidade. Revista Brasileira de Engenharia Agrícola e Ambiental, v.12, n.1, p.3-11, 2008c.

Carvalho, D. F. ; Soares, A. A.; Ribeiro, C. A. A. S.; Sediyama, G. C.; Pruski, F.F. Otimização do uso da água no perímetro irrigado do Gorutuba, utilizando-se a técnica da programação linear. Revista Brasileira de Engenharia Agrícola e Ambiental, v.4, n.2, p.203-209, 2000.

Dantas Neto, J.; Azevedo, C. A. V.; Frizzone, J. A. Uso da programação linear para estimar o padrão de cultura do perímetro irrigado Nilo Coelho. Revista Brasileira de Engenharia Agrícola e Ambiental, v. 1, n.1, p.9-12, 1997.

Ferreira, P. A.; Borges Júnior, J. C. F.; Hedden-Dunkhorst, B.; Andrade, C. L. T. Modelo computacional para suporte à decisão em áreas irrigadas. Parte II: Testes e aplicação. Revista Brasileira de Engenharia Agrícola e Ambiental, v.10, n.4, p.783-791, 2006.

Fox, P.; Rockstrom J.; Barron, J. Risk analysis and economic viability of water harvesting for supplemental irrigation in semi-arid Burkina Faso and Kenya. Agricultural Systems, v.83, n.3, p.231-250, 2005.

Frizzone, J. A. \& Andrade Júnior, A. S. Planejamento de irrigação: análise de decisão de investimento. Brasília: EMBRAPA, 2005, 626p.

Georgiou, P. E.; Papamichail, D. M. Optimization model of an irrigation reservoir for water allocation and crop planning under various weather conditions. Irrigation Science, v.26, n.6, p.487-504, 2008.

Ghahraman, B.; Sepaskhah A. R. Optimal allocation of water from a single purpose reservoir to an irrigation project with pre-determined multiple cropping patterns. Irrigation Science, v.21, n.3, p.127-137, 2002.

Hardaker, J. B.; Huirne, R. B. M.; Anderson, J. R.; Lien, G. Coping with risk in agriculture. 2 ed. London: CABI Publishing, 2004. 352p.

Kale, R. V.; Singh, Rajesh P.; Mahar, P. S. Optimal design of pressurized irrigation subunit. Journal of Irrigation and Drainage Engineering, v.134, n.2, p.137-146, 2008.

Lejano, R. P. Optimizing the layout and design of branched pipeline water distribution systems. Irrigation and Drainage Systems, v.20, n. 1, p.125-137, 2006.

Palisade Corporation, Guide to use @ Risk analysis and simulation add-in for Microsoft Excel. Newfield: Palisade Corporation, 2009. 705p.

Ponciano, N. J.; Souza, P. M.; Mata, H. T. C.; Vieira, J. R.; Morgado, I. F. Análise de viabilidade econômica e de risco da fruticultura na região norte Fluminense. Revista de Economia e Sociologia Rural, v.42, n.4, p.615-635, 2004.

Silva Neto, B.; Stulp, V. J. A consideração de tendências na otimização de sistemas de produção agropecuária sob condições de risco. Revista de Economia e Sociologia Rural, v.38, n.3, p.61-80, 2000.

Tsakiris, G; Spiliotis, M. Cropping pattern planning under water supply from multiple sources. Irrigation and Drainage Systems, v.20, n.1, p.57-68, 2006. 